\title{
Investigation of Nonlinear Granger Causality in the Context of Epilepsy
}

\author{
C. Mahjoub*, J.J. Bellanger†, S. Chaibi*, A. Kachouri* and R. Le Bouquin Jeannès ${ }^{\dagger}$ \\ *National Engineering School of Sfax, LETI Laboratory, Tunisia \\ Email:chahira.mahjoub@enis.tn, sahbi.chaibi@yahoo.fr, abdennaceur.kachouri@enis.rnu.tn \\ 'INSERM U1099 Université de Rennes 1, LTSI Rennes, F-35000, France \\ Email: jean-jacques.bellanger@univ-rennes1.fr, regine.le-bouquin-jeannes@univ-rennes1.fr
}

\begin{abstract}
Granger causality approaches have been widely used to estimate effective connectivity in complex dynamic systems. These techniques are based on the building of predictive models which not only depend on a proper selection of the predictive vectors size but also on the chosen class of regression functions. The question addressed in this paper is the estimation of the model order in the computation of Granger causality indices to characterize the propagation flow between simulated epileptic signals. In this contribution, a new strategy is proposed to select a suitable model order for potentially nonlinear systems. A nonlinear vectorial autoregressive model based on a wavelet network is considered for the identification and an optimal nonlinear model order is selected using the Bayesian information criterion and imported in nonlinear kernel predictors to derive Granger causality. Simulations are firstly conducted on a linear autoregressive model, then on toy nonlinear models and, finally, on simulated intracranial electroencephalographic signals obtained from an electrophysiology based model to reveal the directional relationships between time series data. The performance of our approach proves the effectiveness of the new strategy in the Granger index estimation.
\end{abstract}

Keywords-Epilepsy; Granger causality; Bayesian information criterion; physiology-based model.

\section{INTRODUCTION}

Partial epilepsies have been considered as the most frequent form of drug-resistant seizures which represent roughly $30 \%$ of cases [1]. When antiepileptic drugs do not improve the patients' quality of life, the surgical resection of the epileptogenic zone is a possible treatment to follow. Thus, the region in the brain responsible for seizures should absolutely be localized making the study of the causal relationships among distinct brain areas a necessity. In that context, a variety of approaches have been developed in order to estimate effective connectivity and consequently identify the direction of causal interdependence. Those approaches include measures inspired from information theory such as transfer entropy (TE) [2], phase-related measures such as phase slope index (PSI) [3], and parametric-modeling-based measures such as Granger causality (GC) [4], [5]. TE is a nonlinear method characterized by model-free design (except for observation vectors dimensions) but needs computational capacity [6] whereas PSI is less complex but requires long data segments for available estimation [6]. GC appears as an interesting alternative but needs to consider the model order estimation [6]. The GC approach is mainly based on linear- regression models; however, interactions between or within systems are frequently nonlinear. For this reason, several attempts of nonlinear extensions of GC measures have been proposed including the nonlinear model identification [7], local linear predictors and Kernel Granger Causality [8], [9]. As previously mentioned, the effectiveness of GC algorithms depends on a proper selection of the model order, meaning the maximum time lag to consider so as to get an approximate Markov representation order for the observed time series data. This order can be chosen according to the most commonly used criteria as the Akaike information criterion (AIC), the Bayesian information criterion (BIC) or the cross validation method.

In this study, we propose a new strategy which takes a priori into account possible nonlinear dynamics to accurately choose the model order parameter value. The selected model order is then used for regression functions identification and GC estimation to characterize the directional relationship between time series data.

The remaining of this paper is organized as follows: In Section II, the physiological model used in our study is first introduced, and then the GC approach is briefly reviewed before describing the proposed approach in model order identification and its application to GC approach. Section III is dedicated to experimental results. A linear and two nonlinear models are introduced to ascertain the relevance of the order estimated measure as well as the GC measures obtained for each model. Then, the selected criterion is applied to test GC on intracranial electroencephalographic (iEEG) signals generated by a physiology-based model. Finally, we draw some conclusions in Section IV before suggesting some future perspectives.

\section{MATERIALS AND METHODS}

\section{A. Intracranial EEG signals generation Model}

The physiology-based model was first introduced in [10]. It was used to represent the electrical field activity of a number of distant neuronal populations that might be coupled together. The model produced outputs can be explained as iEEG signals that can be compared to those recorded with proximal electrodes in hippocampus [10]. This type of model, which is currently used in many works in the field of cerebral connectivity, includes nonlinear operators which model thresholding and saturation inherent to action potentials generation. Consequently, it seems a priori interesting in this 
case to test the relevance of the nonlinear approach for causality characterization.

\section{B. Granger Causality}

The GC fundamental concept was derived from Wiener theory before its formalization by Granger in the context of linear Auto-Regressive (AR) modelling of random stochastic processes [4]. Specifically, if the variance of the linear AR prediction error of a first time series, called $X_{1}$, at the present time is decreased by incorporating past measurements from a second time series, called $X_{2}$, then $X_{2}$ can be said to Granger cause $X_{1}$. Thus, the causal influence $X_{2} \rightarrow X_{1}$ can be evaluated by:

$$
G C I_{X_{2} \rightarrow X_{1}}-B=1-\frac{\Gamma_{X_{1} \mid \bar{X}_{1}, \bar{X}_{2}}}{\Gamma_{X_{1} \mid \bar{X}_{1}}}
$$

where $\Gamma_{X_{1} \mid \bar{X}_{1}, \bar{X}_{2}}$ represents the prediction error variance of $X_{1}$ by considering the past values $\bar{X}_{1}$ and $\bar{X}_{2}$ of $X_{1}$ and $X_{2}$ respectively while $\Gamma_{X_{1} \mid \bar{X}_{1}}$ represents the prediction error variance of $X_{1}$ by using only its own past.

If the bivariate index is useful for 2 signals, in case of several signals, the multivariate index is recommended in order to differentiate direct links from indirect ones. Let us consider $Q$ signals recorded simultaneously, the multivariate GC index can be evaluated by:

$$
G C I_{X_{2} \rightarrow X_{1} \mid \bar{X}_{Q-1}}-M=1-\frac{\Gamma_{X_{1} \mid \bar{X}_{1} \ldots \bar{X}_{Q}}}{\Gamma_{X_{1} \mid \bar{X}_{1} \bar{X}_{3} \ldots \bar{X}_{Q}}}
$$

where $\Gamma_{X_{1} \mid \bar{X}_{1} \ldots \bar{X}_{Q}}$ represents the variance of the prediction error of $X_{1}$ by considering the past of all signals and $\Gamma_{X_{1} \mid \bar{X}_{1} \bar{X}_{3} \ldots \bar{X}_{Q}}$ represents the variance of the prediction error of $X_{1}$ by using the past of all signals except $X_{2}$.

A second way to calculate these linear indices can be found in [8], where GC was equivalently reformulated and included a testing statistical procedure SP detailed in [8] to decide if GCI equals zero or not and to handle overfitting, i.e. to eliminate possible parasitic redundancies in the predictive variables set. Both bivariate and multivariate indices are equal to those indicated in Eq. (1) and Eq. (2) respectively, aside from the redundancies correction effect. A generalization of the method was extended to a nonlinear case based on kernel methods [8]. It uses the inhomogeneous polynomial (IP) kernel of order $p$ and the Gaussian kernel of width $\sigma$.

In this paper, the Kernel GC (KGC) method was used since it allows the following benefits [8]:

- detecting effective couplings that cannot be captured by the linear index;

- adapting the nonlinearity of regression model by choosing the adequate kernel function;

- limiting false causality detection rate by a variables selection strategy (through a statistical testing of correlations between the eigenvectors of a Gram matrix and the variable to be predicted).

It should be noted that the linear case is a particular case of the nonlinear one with the IP kernel of an order equal to one. For further details, the reader may refer back to [8], [9].

\section{Proposed approach}

To compute linear or nonlinear Granger indices we have to choose (i) the length of the predictor vectors $\bar{X}_{i}$ which relies on the finite memory depth of a suitable Markovian model for the vectorial observed process, and (ii) the two prediction functions introduced in the Granger indices in a given class of regression functions (linear/nonlinear, kernel based functions, wavelet networks, ...). These two choices can be done jointly by identifying and selecting the order of a linear/nonlinear parametric model thanks to some selection procedures such as AIC or BIC. In this study, we propose to separate the choice of the predictor length and that of the determination of the regression functions by, after identifying an order in a first class of models, selecting the prediction functions in a second class. This allows us to retain the latter class with distinctive characteristics, in this particular case kernel-based regression functions with an interesting selection of significant predictor variables, without being constrained to simultaneously select the size of the arguments of these functions. To summarize, we proceed as follows, (i) we identify from our data a wavelet network nonlinear VAR model as in [7] and select the order using the BIC technique; (ii) we estimate the optimal prediction functions in the class of kernel predictors defined on an Euclidean space whose dimension is equal to the selected order.

The validity of this idea was tested using simulations from different models whose results are reported in Section III. The simulated signals are divided into $N b$ blocks of signals. On each block, the nonlinear model order, denoted by $N L q$, is estimated and then applied to the Granger Causality estimation step. IP kernels with various orders are applied on each data block to deal with more or less nonlinearity strength. It is worth noting that the block length must be chosen in order to ensure the stationarity of signals.

\section{EXPERIMENTS AND RESULTS}

The experiments consist of simulations that were performed 200 times on 2048-point time series for both linear and nonlinear VAR models. In Eq. (3), (4) and (5), the terms $v_{i}(t), i=1,2,3$ represent mutually independent white Gaussian noises with zero means and unit variances. Also, in the following, $q$ denotes the model order obtained from a linear model identification, and $N L q$ denotes the model order obtained from a nonlinear model identification, using AIC or BIC criterion. Simulation is performed with Matlab R2014b and the linear and nonlinear identification are respectively achieved using arx and nlarx functions. For each model the causality test SP was applied to compute the sensitivity and specificity values. 


\section{A. Linear Models}

The stochastic linear model, including both unidirectional and bidirectional causal relationships, was governed by the following equations [11]:

$$
\left\{\begin{array}{l}
x_{1}(t)=0.95 \sqrt{2} x_{1}(t-1)-0.9025 x_{1}(t-2)+v_{1}(t) \\
x_{2}(t)=-0.5 x_{1}(t-1)+0.25 \sqrt{2} x_{2}(t-1)-0.5 x_{3}(t-3)+v_{2}(t) \\
x_{3}(t)=-0.5 x_{1}(t-2)-0.5 x_{2}(t-2)-0.25 \sqrt{2} x_{3}(t-2)+v_{3}(t)
\end{array}\right.
$$

A preliminary step in the GC index computation is the estimation of the model order. In order to evaluate the performance of the proposed method, Table I gives a summary of the number of times the linear and nonlinear orders obtained with the two criteria equal the expected order (i.e. the maximum time lag) over the 200 trials. The maximum lag value is equal to 3 as shown in Eq. (3).

In this linear simulated model, clearly both AIC and BIC are able to estimate correctly the maximum lag by identifying the data to a linear model. Using nonlinear identification of the linear AR model, it is found that the AIC measure remains a reasonable estimator with an estimation accuracy equal to
$95.50 \%$ (let us indicate that the erroneous values of model order obtained in this case are equal to 4) whereas the BIC measure behaves even better as it achieves $100 \%$ of estimation accuracy for the model order.

TABLE I. RESULTS OF THE LINEAR AND NONLINEAR ORDERS ESTIMATED BY THE TWO MEASURES IN THE LINEAR AR SIMULATED MODEL DERIVED FROM EQ. (3)

\begin{tabular}{|c|c|c|}
\hline Information & \multicolumn{2}{|c|}{ Linear Model } \\
\cline { 2 - 3 } Criterion & $q=3$ & $N L q=3$ \\
\hline AIC & $200 / 200$ & $191 / 200$ \\
\hline BIC & $200 / 200$ & $200 / 200$ \\
\hline
\end{tabular}

Given the nonlinear model order estimated by BIC, the statistics (mean and standard deviation) of linear GC values were then computed over the 200 blocks. To better highlight the linearity of time series data, both linear and nonlinear GC indices were evaluated using two values of $p$. The achieved results are displayed in Table II. As expected, performing the two measures on the linear model, we get a stable pattern of connections and one can assume that the interactions between the signals are mainly linear. In both cases, the sensitivity and the specificity are respectively equal to 1 and 0.99 for $p=1$ and to 1 and 0.98 for $p=2$.

TABLE II. RESULTS OF LINEAR GC INDEX (LGC, $P=1$ ) AND NONLINEAR GC INDEX (NLGC, $P=2$ ) FOR THE LINEAR MODEL DESCRIBED BY EQ. (3). THE TABLE INDICATES THE MEANS AND STANDARD DEVIATIONS (STD) FOR BOTH APPROACHES AND THE 6 DIRECTIONS.

\begin{tabular}{|l|l|c|c|c|c|c|c|}
\hline \multicolumn{2}{|c|}{} & $1 \rightarrow 2$ & $2 \rightarrow 1$ & $1 \rightarrow 3$ & $3 \rightarrow 1$ & $2 \rightarrow 3$ & $3 \rightarrow 2$ \\
\hline \multirow{2}{*}{ MEAN } & $L G C$ & 0.4825 & 0 & 0.3500 & $4.88 e-04$ & 0.2837 & 0.3181 \\
\cline { 2 - 8 } & $N L G C$ & 0.4747 & $1.00 e-04$ & 0.3438 & $6.83 e-05$ & 0.2747 & 0.3125 \\
\hline \multirow{2}{*}{ STD } & $L G C$ & 0.0153 & 0 & 0.0162 & $4.87 e-04$ & 0.0167 & 0.0167 \\
\cline { 2 - 8 } & $N L G C$ & 0.0155 & $8.14 e-04$ & 0.0166 & $6.81 e-04$ & 0.0173 & 0.0166 \\
\hline
\end{tabular}

\section{B. Nonlinear models}

For the first nonlinear stochastic toy model, the following multivariate signals were generated [8]

$$
\left\{\begin{array}{l}
x_{1}(t)=0.8\left(1-1.8 x_{1}^{2}(t-1)\right)+0.2\left(1-1.8 x_{2}^{2}(t-1)\right)+0.01 v_{1}(t) \\
x_{2}(t)=1-1.8 x_{2}^{2}(t-1)+0.01 v_{2}(t) \\
x_{3}(t)=0.8\left(1-1.8 x_{3}^{2}(t-1)\right)+0.2\left(1-1.8 x_{1}^{2}(t-1)\right)+0.01 v_{3}(t)
\end{array}\right.
$$

The numbers of trials (among the 200 trials) for which the obtained linear and nonlinear orders with the two criteria are equal to the expected order are depicted in Table III and prove the efficiency of our proposed approach.

TABLE III. RESULTS OF THE LINEAR AND NONLINEAR ORDERS ESTIMATED BY THE TWO MEASURES IN THE NONLINEAR AR MODEL DESCRIBED BY EQ. (4)

\begin{tabular}{|c|c|c|}
\hline $\begin{array}{c}\text { Information } \\
\text { Criterion }\end{array}$ & \multicolumn{2}{|c|}{ First nonlinear Model } \\
\cline { 2 - 3 } & $q=1$ & $N L q=1$ \\
\hline $\mathrm{AIC}$ & $0 / 200$ & $200 / 200$ \\
\hline & & \\
\hline $\mathrm{BIC}$ & $0 / 200$ & $200 / 200$ \\
\hline
\end{tabular}

The maximum lag value is equal to 1 as shown in Eq. (4). As expected, using the linear identification of the nonlinear system could lead to some erroneous model order estimation.
In fact, as displayed in Table III, the model order using linear model identification (denoted by $q$ ) is not correctly estimated. The estimated orders using AIC and BIC are then in the range [6-14] and [4-6] respectively. In contrast, using the nonlinear identification approach, both criteria accurately estimate the maximum lag value (denoted by $N L q$ ) which is equal to 1 .

As previously indicated, the nonlinear order estimated by the BIC measure was retained afterwards, and the means and the standard deviations of the corresponding linear and nonlinear GC values over the 200 trials are given in Table IV. It is obvious that, according to Table IV, the linear GC index fails to detect the two significant links $(2 \rightarrow 1$ and $1 \rightarrow 3$ ) since the index values are all close to zero. However, performing the nonlinear $\mathrm{GC}$, with a kernel order equal to 2, reveals both influences $2 \rightarrow 1$ and $1 \rightarrow 3$ as the index values are respectively equal to 0.9919 and 0.9879 . In addition, the linear GC scores lowest in sensitivity and specificity compared to the nonlinear GC, these quantities being respectively equal to 0.73 and 0.71 for $p=1$ and to 1 and 0.99 for $p=2$.

For the second nonlinear stochastic toy model [12], the multivariate signals with both linear and nonlinear interactions were generated using Eq. (5) 
TABLE IV. RESUlTS OF LINEAR GC INDEX (LGC, $P=1$ ) AND NONLINEAR GC INDEX (NLGC, $P=2$ ) FOR THE FIRST NONLINEAR MODEL DESCRIBED BY EQ. (4). THE TABLE INDICATES THE MEANS AND STANDARD DEVIATIONS (STD) FOR BOTH APPROACHES AND THE 6 DIRECTIONS.

\begin{tabular}{|c|c|c|c|c|c|c|c|}
\hline \multicolumn{2}{|c|}{} & $1 \rightarrow 2$ & $2 \rightarrow 1$ & $1 \rightarrow 3$ & $3 \rightarrow 1$ & $2 \rightarrow 3$ & $3 \rightarrow 2$ \\
\hline \multirow{3}{*}{ MEAN } & $L G C$ & 0.0046 & 0.0146 & 0.0028 & 0 & 0.0019 & $8.57 e-04$ \\
\cline { 2 - 8 } & $N L G C$ & $1.68 e-05$ & 0.9919 & 0.9879 & $2.47 e-05$ & $5.22 e-05$ & $1.10 e-04$ \\
\hline \multirow{3}{*}{ STD } & $L G C$ & 0.0029 & 0.0032 & 0.0034 & 0 & 0.0028 & $6.16 e-04$ \\
\cline { 2 - 8 } & $N L G C$ & $5.21 e-04$ & 0.0013 & 0.0014 & $3.49 e-04$ & $5.21 e-04$ & $7.87 e-04$ \\
\hline
\end{tabular}

$$
\left\{\begin{aligned}
x_{1}(t)= & 3.4 x_{1}(t-1)\left(1-x_{1}^{2}(t-1)\right) \mathrm{e}^{-x_{1}^{2}(t-1)}+0.4 v_{1}(t) \\
x_{2}(t)= & 3.4 x_{2}(t-1)\left(1-x_{2}^{2}(t-1)\right) \mathrm{e}^{-x_{2}^{2}(t-1)}+0.5 x_{1}(t-1) x_{2}(t-1) \\
& +0.4 v_{2}(t) \\
x_{3}(t)= & 3.4 x_{3}(t-1)\left(1-x_{3}^{2}(t-1)\right) \mathrm{e}^{-x_{3}^{2}(t-1)}+0.3 x_{2}(t-1) \\
& +0.5 x_{1}^{2}(t-1)+0.4 v_{3}(t)
\end{aligned}\right.
$$

The direct causal relationship implemented in Eq. (5) are $1 \rightarrow 2,1 \rightarrow 3$ and $2 \rightarrow 3$. The orders estimated by the linear and nonlinear techniques are displayed in Table $\mathrm{V}$.

In this experiment, we note that the AIC measure behaves worse in the linear case. As a matter of fact, all other estimated orders are equal to 1 over the 200 simulations $(100 \%)$ whereas only 30 estimated orders assuming linear model and using AIC are correct.

TABLE V. RESULTS OF THE LINEAR AND NONLINEAR ORDERS ESTIMATED BY THE TWO MEASURES IN THE NONLINEAR AR MODEL DESCRIBED BY EQ. (5)

\begin{tabular}{|c|c|c|}
\hline Information & \multicolumn{2}{|c|}{ Second nonlinear Model } \\
\cline { 2 - 3 } Criterion & $q=1$ & $N L q=1$ \\
\hline AIC & $30 / 200$ & $200 / 200$ \\
\hline BIC & $200 / 200$ & $200 / 200$ \\
\hline
\end{tabular}

As previously mentioned, we retained the $N L q$ value estimated by the BIC criterion to compute the multivariate GC indices. GC measures using the IP kernel with different values of $p$ are displayed in Fig. 1. Obviously, the linear GC fails to detect the flows $1 \rightarrow 2$ and $1 \rightarrow 3$ which both display nonlinearities and only detects the linear influence $2 \rightarrow 3$. Applying the nonlinear GC with various kernel values $(p \geq 2)$, the different implemented causal relationships are detected and a stable connection pattern can be revealed. The results obtained in the three opposite directions $2 \rightarrow 1,3 \rightarrow 1$ and $3 \rightarrow 2$ all lead to extremely low index measures whatever the value of $p$. Let us indicate that the linear GC has a low score in sensitivity $(0.34)$ whereas increasing the value of $p$ makes the sensitivity score equal to 1 . As for specificity, it obtains high scores in most cases (between 0.89 and 0.98 ).

\section{Physiology-based model}

In a third step, we considered a physiology-based model with unidirectional causality from a first epileptic population (population 1) to a second one (population 2). In this model, we generated 400 -s signals with a sampling frequency equal to $512 \mathrm{~Hz}$. After data acquisition, each signal was divided into 100 blocks of 2048 points. Thus each block corresponded to a 4-s length. This causal relationship was expected since, when removing the 'physical' directional link between the first population and the second one (characterized by a connectivity parameter that we set to zero in the model) a dramatic modification of the dynamics of the EEG signal simulated by the second population was observed. Now, at first glance, measures of directional connectivity derived from linear Granger Causality could not face this issue and led to low values. Inasmuch as nonlinear operators were introduced in each population model, it seemed interesting to supply these first measures with measures of nonlinear Granger Causality.

According to the previous results of model order selection, only the order $N L q$ obtained by BIC measure was used. Fig. 2 indicates the box plots of the GC values computed on 100 blocks of time series using different IP kernel order values. The result obtained with $p=1$ shows that the value of the index for the direction $1 \rightarrow 2$ tends to be significantly greater than that for the direction $2 \rightarrow 1$.When statistically analyzing the 100 blocks of samples to compare the statistical index distributions for $1 \rightarrow 2$ and $2 \rightarrow 1$ using the 2 populations Kolmogorov-Smirnov test (kstest2 in matlab) the obtained pvalue is equal to $2.63 e-46$. These results do not contradict the ground truth, i.e. a directional influence from population 1 to population 2. However, the measured GC index values remain small in the expected direction. Let us indicate that the estimated model orders we obtained were in the range of [3-6].

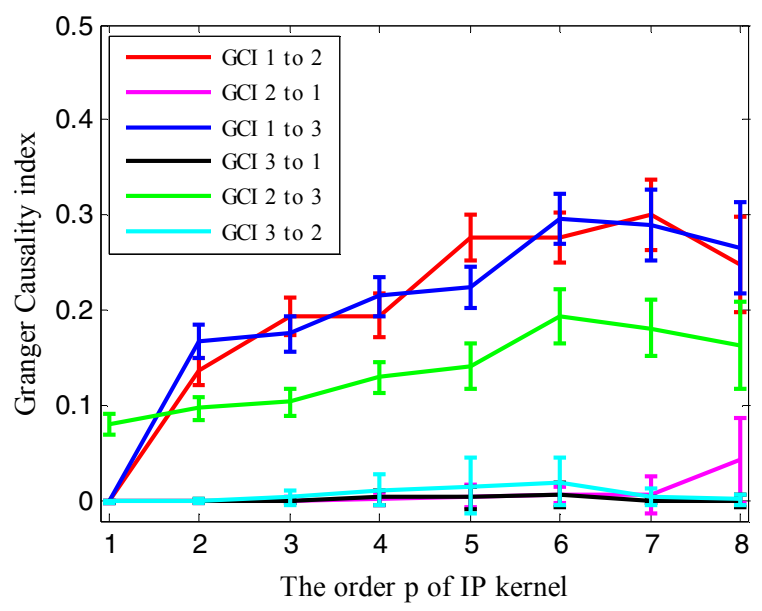

Fig. 1. The causal relationships between all pairs of signals using linear and nonlinear multivariate $\mathrm{GC}$ with various kernel values. The value $p=1$ corresponds to the linear case.

Using the nonlinear GC approach with an IP kernel (or a Gaussian kernel not presented here) does not result in a noticeable increasing of the measured Granger indices compared to the linear kernel. For the two nonlinear orders, the p-values are respectively $1.07 e-44$ and $2.70 e-11$. Hence the results with $p=3$ do not bring information 
improvement. The sensitivity for $p=1, p=2$ and $p=3$ is respectively equal to $0.96,0.90$ and 0.45 . We can note a strong sensitivity decrease with $p=3$ compared to $p=2$. Let us indicate that the specificity slightly increases with the value of $p$ (from 0.92 for $p=1$ to 0.98 for $p=3$ ). Different assumptions could be made relying on these results, either both IP and Gaussian kernels are not sensitive to the kind of nonlinearity introduced in this physiological model, or, even if our system is nonlinear and its block representation could make us expect a (soft) nonlinear influence, the optimal prediction functions could be approximately linear. We can speculate that the large modification of the second population dynamics, when the input of this population is fed by the output of the first one, could be mainly explained by an influence of the (positive) temporal mean value of the first population output. This component induces moving of the stable fixed point in the second population state space and a modification of the linearized model. This explains the observed dynamics modification which, in this case, could not be explained by a strong influence of the centered component of the first population output (the signal minus its temporal mean level). As Granger causality is sensitive to only this time variable component, its value remains low. We tested this conjecture by replacing the first population output by its mean value, and we observed the same dramatic changes in the second population dynamics. These conclusions could impact the interpretation of causality influences between neural populations when dealing with this type of model.

A question of great interest could be to estimate GC index using the predictors derived from the wavelet identification (WI). In fact, we have also tested this solution but we do not present any detailed results in this contribution. As a matter of fact, the WI approach fails sometimes to detect causal links and especially in systems including both linear and nonlinear interactions as those introduced in Eq. (5). For instance, when we tested the WI method in such a situation, the flow $2 \rightarrow 3$ was not detected. These reasons led us to only develop the KGC approach.

\section{CONCLUSION}

In this study, to estimate linear/nonlinear Granger causality we proposed a two-step strategy that (i) determines the common length of the predictor vectors by estimating the Markov memory depth of the observed vector random process through a nonlinear AR identification and a BIC or AIC procedure and (ii) establishes the two linear/nonlinear prediction functions used in the Granger indices calculation following the kernel approach. The results we reported are in favor of the BIC measure which is the best choice even when the data are provided by a linear model.

This approach allows us to better interpret the causal influence emerging from a widely used model of coupled neural populations. According to these results, real epileptic signals must be now considered to determine the organization of the epileptogenic zone, the preeminent difficulty for validation being to get a reliable ground truth from the clinician on the effective cerebral connectivity. Furthermore, optimization steps have to be considered such as the investigation of other kernels to detect other kinds of nonlinearity which could not be detected by IP and/or Gaussian kernels.

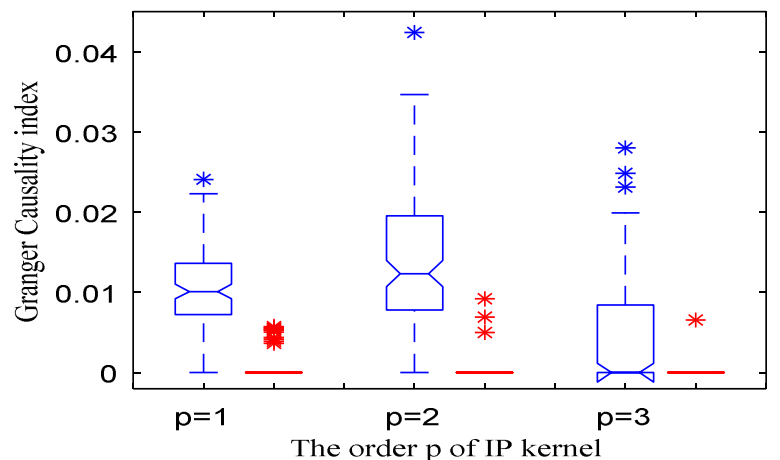

Fig. 2. Box plots of GC index for the physiological model using various IP kernel values. For each value of $p$, the box plots are displayed for the information flow from 1 to 2 (left-hand side) and from 2 to 1 (right-hand side). The null interval between the first and last quartiles obtained for the latter direction can be explained by the thresholding rule introduced to eliminate redundant predictors in kernel Granger method (this leads to a Granger index value equal to zero when none of the linear or nonlinear functions of $\bar{X}_{2}$ implicitly introduced as features in the kernel approach is significantly correlated with the predicted variable).

\section{REFERENCES}

[1] J Epilepsy: Fact Sheet: World Health Organization, Available at: http://www.who.int/mediacentre/factsheets/fs999/en/, 2017.

[2] X. Mao and P. Shang, "Transfer entropy between multivariate time series," Commun. Nonlinear Sci. Numer. Simul., vol. 47, pp. 338-347, 2017.

[3] G. Nolte, A. Ziehe, V. V. Nikulin, A. Schlögl, N. Krämer, T. Brismar, and K. R. Müller, "Robustly estimating the flow direction of information in complex physical systems," Phys. Rev. Lett., vol. 100, no. 23, pp. 14, 2008.

[4] Granger, C. W. J., "Investigating causal relations by econometric models and cross-spectral methods," Econometrica, vol. 37, no. 3, pp. 424-438, Aug, 1969.

[5] E. Siggiridou and D. Kugiumtzis, "Granger Causality in Multi-variate Time Series using a Time Ordered Restricted Vector Autoregressive Model," IEEE Trans. Signal Process., vol. 64, no. 7, 2016.

[6] M. J. Silfverhuth, H. Hintsala, J. Kortelainen, and T. Seppänen, "Experimental comparison of connectivity measures with simulated EEG signals," Med. Biol. Eng. Comput., vol. 50, no. 7, pp. 683-688, 2012.

[7] L. Faes, G. Nollo, and K. Chon, "Assessment of Granger Causality by Nonlinear Model Identification: Application to Short-term Cardiovascular Variability," Ann. Biomed. Eng., vol. 36, no. 3, pp. 381395, 2008.

[8] Marinazzo, M. Pellicoro, and S. Stramaglia, "Kernel-Granger causality and the analysis of dynamical networks," pp. 1-9, 2008.

[9] W. Liao, D. Marinazzo, Z. Pan, Q. Gong, and H. Chen, "Kernel Granger Causality Mapping Effective Connectivity on fMRI Data," vol. 28, no. 11, pp. 1825-1835, 2009.

[10] F. Wendling, A. Hernandez, J. J. Bellanger, P. Chauvel, and F. Bartolomei, "Interictal to Ictal Transition in Human Temporal Lobe Epilepsy: Insights From a Computational Model of Intracerebral EEG," J. Clinical Neurophysiol., vol. 22, no. 5, pp. 343-356, 2005.

[11] C. Yang, "Contribution to effective connectivity analysis in epilepsy," $\mathrm{Ph}$. D. Dissertation, University of Rennes, 2012.

[12] B. Gourévitch and R. Le Bouquin Jeannes, "Linear and nonlinear causality between signals: methods, examples and neurophysiological applications," Biol. Cybern., vol. 1, pp. 349-369, 2006. 